\title{
Preliminary analysis of miRNA pathway in Schistosoma mansoni
}

\author{
Matheus S. Gomes ${ }^{\text {a,* }}$, Fernanda J. Cabral ${ }^{\text {b }}$, Liana K. Jannotti-Passos ${ }^{c}$, Omar Carvalho ${ }^{\text {, }}$, Vanderlei Rodrigues ${ }^{\text {b }}$, \\ Elio H. Baba a , Renata G. Sá a,* \\ a Núcleo de Pesquisas em Ciências Biológicas (NUPEB), Universidade Federal de Ouro Preto, Morro do Cruzeiro, 35400-000, Ouro Preto, MG, Brazil \\ ${ }^{\mathrm{b}}$ Faculdade de Medicina de Ribeirao Preto, Universidade de Sao Paulo, Brazil \\ c Centro de Pesquisas Rene Rachou, FIOCRUZ, Belo Horizonte, MG, Brazil
}

\section{A R T I C L E I N F O}

Article history:

Received 19 August 2008

Received in revised form 30 September 2008

Accepted 16 October 2008

Available online 28 October 2008

\section{Keywords:}

MicroRNAs

Post-transcriptional gene silencing

Schistosoma mansoni

Gene expression

qRT-PCR Real Time

\begin{abstract}
A B S T R A C T
RNA silencing refers to a series of nuclear and cytoplasmatic processes involved in the post-transcriptional regulation of gene expression or post-transcriptional gene silencing (PTGS), either by sequence-specific mRNA degradation or by translational arrest. The best characterized small RNAs are microRNAs (miRNAs), which predominantly perform gene silencing through post-transcriptional mechanisms. In this work we used bioinformatic approaches to identify the parasitic trematode Schistosoma mansoni sequences that are similar to enzymes involved in the post-transcriptional gene silencing mediated by miRNA pathway. We used amino acid sequences of well-known proteins involved in the miRNA pathway against S. mansoni genome and transcriptome databases identifying a total of 13 putative proteins in the parasite. In addition, the transcript levels of SmDicer1 and SmAgo2/3/4 were identified by qRT-PCR using cercariae, adult worms, eggs and in vitro cultivated schistosomula. Our results showed that the SmDicer1 and SmAgo2/3/4 are differentially expressed during schistosomula development, suggesting that the miRNA pathway is regulated at the transcript level and therefore may control gene expression during the life cycle of $S$. mansoni.
\end{abstract}

(C) 2008 Published by Elsevier Ireland Ltd.

\section{Introduction}

Post-transcriptional gene silencing (PTGS) or RNA silencing is a complex mechanism involving conserved proteins and at least two distinct pathways. These are known as the siRNAs (shorts interfering RNAs) and miRNAs (microRNAs) pathways involved in the silencing of endogenous or exogenous mRNAs each with differing specificity but with several overlapping components [1]. These RNAs were initially identified as complex structures of dsRNAs, processed in mature small RNAs of 17 to 25 nt in size, which play a role on cleavage or inhibition of specific translation of mRNAs. siRNAs are the cleavage products of longer dsRNAs that are generated by RNA-dependent RNA polymerases, or from bidirectional transcription of genes or transposable elements. miRNAs mediate translational repression, although, in some cases, they can also direct mRNA degradation. The isolation and characterization of diverse genes that codify components of the microRNAs pathway in Arabidopsis thaliana, Caenorhabditis elegans, Drosophila melanogaster and Homo sapiens support the hypothesis

Abbreviations: ESTs, Expressed Sequence Tag; PTGS, post-transcriptional gene silencing; siRNAs, shorts interfering RNAs; miRNAs, microRNAs; dsRNAs, doublestranded RNA; MTS, mechanically transformed schistosomula; cDNA, complementary DNA; PCR, polymerase chain reaction.

* Corresponding authors. Gomes is to be contacted at Tel.: +55 31 35591698; fax: +55 3135591680.

E-mail address: matheusop@yahoo.com.br (M.S. Gomes). that the silencing of RNA constitutes a well conserved system found in different organisms [2-5].

The machinery of RNA silencing mediated by miRNA is dependent on several proteins [6]. In the initiation stage, the primary miRNA (primiRNA) is transcribed by a type II RNA polymerase. Inside the nucleus the pri-miRNA is cleaved into pre-miRNA that is an ATP-dependent microprocessor complex coordinated by RNase III Drosha [7]. The premiRNA, which exhibits a cramp-like structure and contains approximately 60-70 nt, is transported from the nucleus to the cytoplasm by the complex Ran-GTP/Exportina-5 [8,9]. Dicer and Argonaute consist of two core proteins involved in the silencing of RNA [6]. In the cytoplasm the pre-miRNA is cleaved by Dicer generating the mature miRNA. The latter is presented to the RNA induced silencing complex (RISC) which in D. melanogaster is composed of the main constituents: Argonaute, Tudor-SN, Fmr1 and Vig [10]. Different Argonaute paralogs were identified in various organisms where they give rise to different forms of RISC, each playing a distinct role [11-14].

Dicer is a ribonuclease in the RNase III family that contains two RNase III domains and one PAZ domain. A main feature of the Argonaute proteins is the presence of PAZ and PIWI domains in their structure [15]. The PIWI domain functions by directing the dsRNA to hydrolysis and it is structurally similar to a domain found in the RNAse H. The PAZ domain (Piwi/Argonaute/Zwile) identified in proteins Argonaute and Dicer, consists of 130 amino acids and it coordinates the interaction between the two proteins forming a heterodimer $[16,17]$. 
Table 1

Putative miRNA pathway sequences in Schistosoma mansoni

\begin{tabular}{|c|c|c|c|c|c|c|c|}
\hline miRNA pathway & $\begin{array}{l}\text { S. mansoni } \\
\text { putative } \\
\text { orthologs }\end{array}$ & Function & Domains and Pfam number & $\begin{array}{l}\text { Identity with } \\
\text { D. melanogaster (\%) }\end{array}$ & $\begin{array}{l}\text { D. melanogaster } \\
\text { orthologs (FlyBase) }\end{array}$ & $\begin{array}{l}\text { C. elegans } \\
\text { orthologs } \\
\text { (WormBase) }\end{array}$ & $\begin{array}{l}\text { S. japonicum } \\
\text { putative } \\
\text { orthologs }\end{array}$ \\
\hline \multicolumn{8}{|l|}{ Endoribonuclease } \\
\hline Dicer & Smp_169750 (SmDicer1) & $\begin{array}{l}\text { Processing of } \\
\text { miRNA precursor }\end{array}$ & $\begin{array}{l}\text { dsRNA_bind (PF03368); } \\
\text { PAZ (PF02170); } \\
\text { Ribonuclease_3 (PF00636) }\end{array}$ & 47 & FBgn0039016 & WBGene00000939 & SJCHGC08817 \\
\hline Drosha & $\begin{array}{l}\text { Smp_142510.2 (SmDrosha2) } \\
\text { Smp_142510.1 (SmDrosha1) }\end{array}$ & $\begin{array}{l}\text { Processing of } \\
\text { primary miRNA } \\
\text { transcripts }\end{array}$ & $\begin{array}{l}\text { Ribonuclease_3 (PF00636); } \\
\text { dsrm (PF00035) }\end{array}$ & $\begin{array}{l}46 \\
48\end{array}$ & FBgn0026722 & WBGene00009163 & SJCHGC08309 \\
\hline \multicolumn{8}{|c|}{ Component of RISC } \\
\hline Argonaute & $\begin{array}{l}\text { Smp_140010 (SmAgo1) } \\
\text { Smp_179320 (SmAgo2) } \\
\text { Smp_102690.2 (SmAgo3) } \\
\text { Smp_102690.3 (SmAgo4) }\end{array}$ & Short RNA binding & $\begin{array}{l}\text { DUF1785 (PF08699); } \\
\text { PAZ (PF02170); } \\
\text { Piwi (PF02171) }\end{array}$ & $\begin{array}{l}68 \\
33 \\
29 \\
33\end{array}$ & $\begin{array}{l}\text { FBgn0026611 } \\
\text { FBgn0046812 }\end{array}$ & $\begin{array}{l}\text { WBGene00000105 } \\
\text { WBGene00000106 }\end{array}$ & $\begin{array}{l}\text { SJCHGC07755 } \\
\text { SJCHGC07884 } \\
\text { SJCHGC01111 } \\
\text { SJCHGC05069 } \\
\text { SJCHGC07014 } \\
\text { SJCHGC02502 }\end{array}$ \\
\hline Tudor-SN & Sm01663 & Nuclease & $\begin{array}{l}\text { SNase (PF00565); } \\
\text { TUDOR (PF00567) }\end{array}$ & 36 & FBgn0035121 & WBGene00006626 & $\begin{array}{l}\text { SJCHGC09149 } \\
\text { SJCHGC04700 } \\
\text { SJCHGC05245 }\end{array}$ \\
\hline Fmr1 & Smp_099630 & RNA binding & KH_1 (PF00013) & 25 & FBgn0028734 & - & SJCHGC09283 \\
\hline \multicolumn{8}{|l|}{ Other factors } \\
\hline Partner Drosha & Smp_087220 & RNA binding & dsrm (PF00035) & 29 & FBgn0039861 & WBGene00011908 & SJCHGC09172 \\
\hline Partner Dicer & Smp_023670 & RNA binding & dsrm (PF00035) & 33 & FBgn0032515 & WBGene00017025 & - \\
\hline Exportin-5 & $\begin{array}{l}\text { Smp_152800.1 } \\
\text { Smp_152800.2 }\end{array}$ & $\begin{array}{l}\text { Nuclear export of } \\
\text { miRNA precursors }\end{array}$ & Xpo1 (PF08389); & $\begin{array}{l}21 \\
21\end{array}$ & FBgn0031051 & - & $\begin{array}{l}\text { SJCHGC09381 } \\
\text { SJCHGC05562 }\end{array}$ \\
\hline
\end{tabular}

In Schistosoma mansoni the silencing mechanism mediated by posttranscriptional modification was suggested by Blanton and Licate [18] as part of the hypothesis that during the cercariae to schistosomula transformation there is a block in translation. Based on this statement we used bioinformatic approaches to identify miRNA pathway components in this parasite. In addition, we performed quantitative Real-Time PCR of two key proteins of the pathway, Dicer and Argonaute, to determine the relative levels of mRNA in adult worms, cercariae, eggs and mechanically transformed schistosomula cultivated from $3.5 \mathrm{~h}$ to 3 days. Even though our study has evidenced the presence of these transcripts in all investigated stages we highlight its importance for post-transcriptional control during the cercariae to schistosomula transition.

\section{Materials and methods}

\subsection{Sequence retrieving of the miRNA components pathway}

Sequences involved in the miRNA pathway were searched in S. mansoni genome database version 4.0 from GeneDB (http://www. genedb.org/genedb/smansoni/) and S. mansoni transcriptome project (http://verjo18.iq.usp.br/schisto/) using amino acid sequences of D. melanogaster and C. elegans orthologs as queries. The BLASTp algorithm, underpinned by the Pfam (v22.0) and CDD databases was used for searches of conserved protein domains or motifs from S. mansoni sequences.

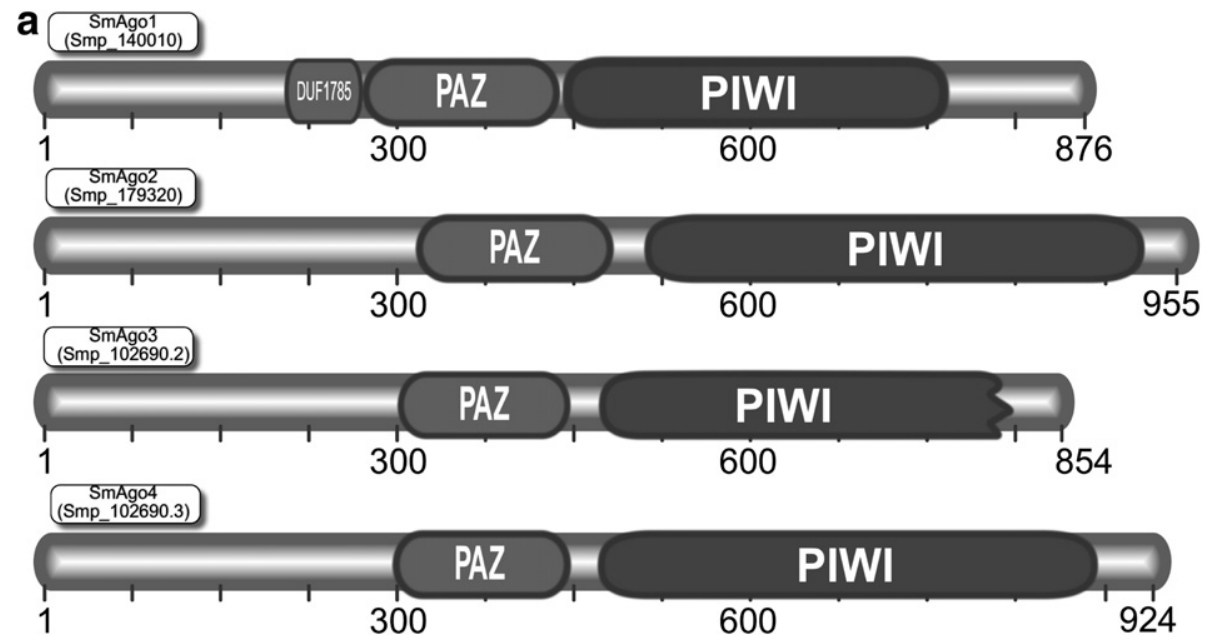

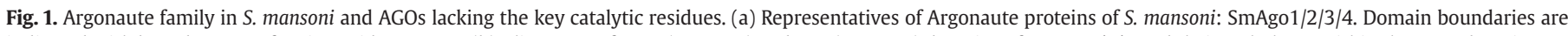

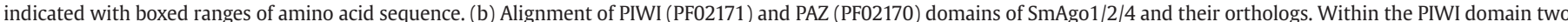

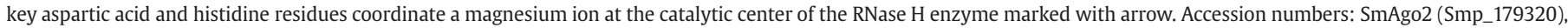

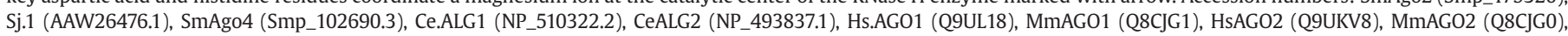
DmAgo1 (NP_725342), SmAgo1 (Smp_140010), At.AG01 (AAB91987.1), and AtAG09 (CAD66636.1). 


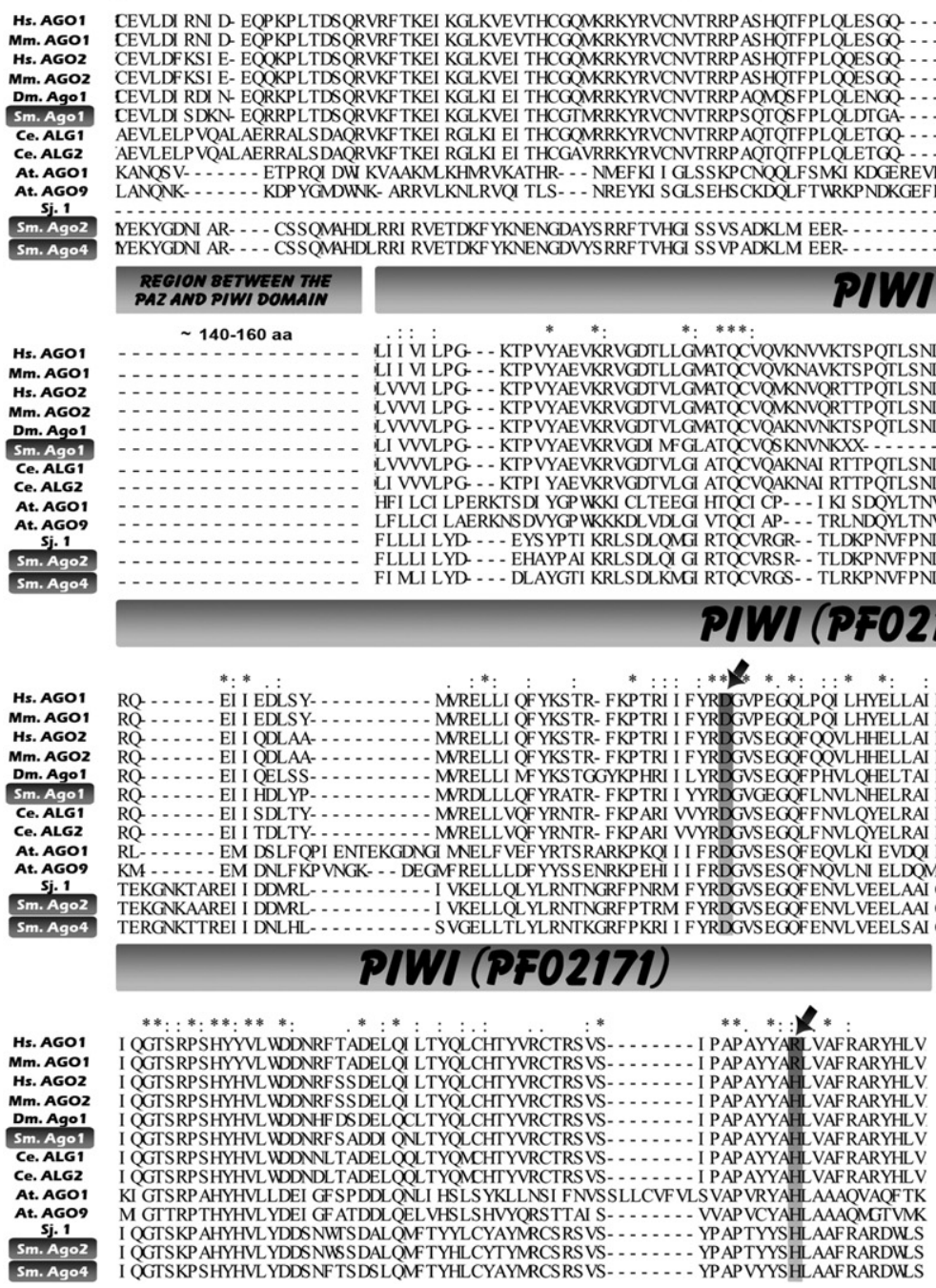

Fig. 1 (continued).

\subsection{Alignment and phylogenetic analyses}

Multiple alignment of SmDicer1 and SmAgo1/2/3/4 were performed by ClustalX 2.0 and phylogenetic analyses were conducted in MEGA 4 [19]. Phylogenetic tree of these sequences were inferred using the Neighbor-Joining method [20]. The bootstrap consensus tree inferred from 1000 replicates was used to represent the evolutionary history of the taxa analyzed. Branches corresponding to partitions reproduced in less than $50 \%$ bootstrap replicates are collapsed. The percentage of replicate trees in which the associated taxa clustered together in the bootstrap test (1000 replicates) is shown next to the branches [21]. The tree was drawn to scale, with branch lengths in the same units as those of the evolutionary distances used to infer the phylogenetic tree. All positions containing gaps and missing data were eliminated from the dataset.

\subsection{Parasites}

S. mansoni LE strain was maintained by routine passage through Biomphalaria glabrata snails and BALB/c mice. The infected snails were induced to shed cercariae under light exposure for $2 \mathrm{~h}$ and the cercariae were recovered by sedimentation on ice. Adult worm parasites were obtained by liver perfusion of mice after 50 days of infection. Mechanically transformed schistosomula (MTS) were prepared as described by Harrop and Wilson [22]. Briefly, cercariae were recovered, and washed in RPMI 1640 medium (Invitrogen), before vortexing at maximum speed for $90 \mathrm{~s}$ and immediately cultivated during $3.5 \mathrm{~h}$ at $37{ }^{\circ} \mathrm{C}, 5 \% \mathrm{CO}_{2}$. Then the recovered schistosomula were washed with RPMI 1640 until no tails were detected. For subsequent incubations, the parasites were maintained in M169 medium supplemented with $10 \%$ FBS, penicillin and streptomycin $(100 \mu \mathrm{g} / \mathrm{mL})$ and $5 \%$ of Schneider's medium [23] at $37^{\circ} \mathrm{C}$ on $5 \%$ of $\mathrm{CO}_{2}$ during $8.5 ; 18.5$; 24; 48 and $72 \mathrm{~h}$.

\subsection{RNA preparation and quantitative Real Time $P C R$}

Total RNA was obtained using a combination of the Trizol reagent (GIBCO-BRL) and chloroform for extraction, and then purified on column using the "Purelink Micro-to-Midi Total RNA purification system" (Invitrogen). The preparation was treated with RNase-free DNase I in 3 different rounds by decreasing enzyme concentration (RQ1 DNase; Promega, Belo Horizonte, Brazil). RNA was quantified using a spectrophotometer and $5 \mu \mathrm{g}$ reverse transcribed using an oligodT primer from the Thermoscript RT-PCR System (Invitrogen São Paulo, Brazil) as described by the manufacturer. The cDNAs encoding SmAgo2/3/4 and SmDicer1 were obtained by PCR amplification using the forward primer $5^{\prime}$-TCACGCACGGTAGATCAGG-3' and reverse primer 5'-AGGTCCGCGTCAATTTGG-3' for SmAgo2/3/4 and the forward 5'-TCTTCCGTCCACCATTCG-3' and reverse 5'-TGCCAACAACACAATTCC3' for SmDicer1. The efficiency of DNAse I treatment was evaluated by 
PCR amplification of the CDNA reaction mix without the addition of the Thermoscript enzyme. All the primers were designed using the program GeneRunner ${ }^{\circledR}$. All PCR products were sequenced in the ABI 3100 automated sequencer (Applied Biosystems) using the Dye terminator kit. Reverse-transcribed cDNA samples were used as templates for PCR amplification using SYBR Green Master Mix UDG-ROX ${ }^{\circledR}$ (Invitrogen) and 7500 Real Time PCR System (Applied Biosystems). Specific primers for $S$. mansoni $\alpha$-tubulin were used as an endogenous control (GenBank access no. M80214) [24] (forward 5'-CGTATTCGCAAGTTGGCTGACCA-3', reverse 5'-CCATCGAAGCGCAGTGATGCA-3'). The efficiency for each pair of primers was evaluated according to the protocol developed by the Applied Biosystems application (cDNA dilutions were $1: 10,1: 100$ and 1:1000). For both investigated transcripts three biological replicates were performed and their gene expression normalized against the $\alpha$-tubulin transcript according to the $2^{-\Delta \Delta \mathrm{Ct}}$ method [25] using the Applied Biosystems 7500 software.

\section{Results}

3.1. Data mining of miRNA pathway related sequences in S. mansoni databases and analyses of their putative domains

The primary goal of our study was to use bioinformatic tools for an in silico reconstitution of the miRNA pathway in $S$. mansoni. Our analyses revealed that the members of this pathway are wellconserved at the amino acid level when compared to their orthologs found in diverse organisms such as D. melanogaster, C. elegans,
H. sapiens, Mus musculus and A. thaliana. Due to the high representation of members of this pathway in D. melanogaster we decided to evaluate their degree of similarity with the related $S$. mansoni sequences (Table 1). When comparing between the S. mansoni predicted genes and their related ESTs from $S$. japonicum up to $70 \%$ sequence identity could be found.

Based on our database mining we suggest that $S$. mansoni contains four members of Argonaute proteins termed SmAgo1, SmAgo2, SmAgo3 and SmAgo4 containing 876, 955, 854 and 924 amino acid residues, respectively (Fig. 1a). Alignments between SmAgo2/3/4 proteins revealed $58 \%$ identity and $17 \%$ between SmAgo 1/2/3/4. When comparing the degree of identity of SmAgo1/2/3/4 proteins with their orthologs in D. melanogaster we observed 68, 33, 29 and 33\%, respectively (Table 1 ). Moreover, the alignment of AGO-like members revealed that most of their components, including SmAgo1/2/4 exhibited the conservation of key amino acid $(\mathrm{D} / \mathrm{D} / \mathrm{H})$ residues that coordinate the $\mathrm{Mg}^{2+}$ ion at the PIWI domain (Fig. 1b).

We also identified three putative RNase III proteins, including SmDicer1, SmDrosha1 and SmDrosha2 containing 2174, 1531 and 1577 amino acid residues, respectively (Fig. 2a). Furthermore, we can clearly observe the four catalytic residues ED and DE of endoND 1/2 domains of SmDicer1 and SmDrosha1/2 (Fig. 2b).

\subsection{Phylogenetic relationship among Argonautes and RNAse III family protein members}

The phylogenetic tree created with the Neighbor-Joining method was used to separate the putative SmAgo1/2/3/4, SmDicer1 and

a
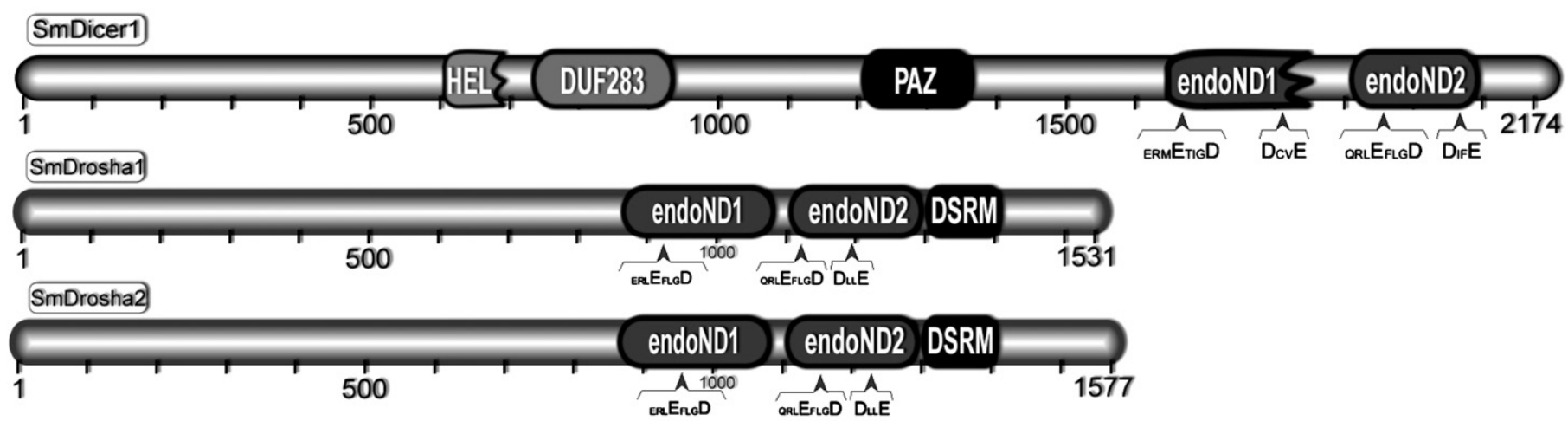

\begin{tabular}{|c|c|c|}
\hline $\begin{array}{c}\text { RNAse III } \\
\text { family }\end{array}$ & endoND1 & endoND2 \\
\hline \multirow{2}{*}{$\begin{array}{l}\text { Dicer1 } \\
\text { Hs.Dicer1 }\end{array}$} & & \\
\hline & ERLE $\mathbf{E}_{M L G} \mathbf{D}_{\ldots . . . . .} \mathbf{D}_{\mathrm{CV}} \mathbf{E}$ & 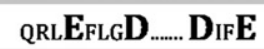 \\
\hline \multirow{3}{*}{$\begin{array}{l}\text { Dm.Dicer1 } \\
\text { Ce.DCR1 } \\
\text { SmDicer1 }\end{array}$} & $\mathrm{ERL}_{\mathrm{TIG}} \mathbf{D} \ldots \ldots . . . \mathbf{D}_{\mathrm{cV}} \mathbf{E}$ & $\mathrm{QRL}_{\mathrm{RLG}} \mathbf{D}_{\ldots \ldots . .} \mathbf{D}_{\mathrm{VF}} \mathbf{E}$ \\
\hline & ${ }_{\text {ERFETIG }}$....... D $_{A V E}$ & QRLEFLGD...... DIFE \\
\hline & ERMETIG D D D & $\mathrm{QRL}_{\mathrm{E} L \mathrm{~F}} \mathbf{D}_{\ldots \ldots . .}$ D $_{\mathrm{IF}} \mathbf{E}$ \\
\hline \multirow{2}{*}{$\begin{array}{c}\text { Drosha } \\
\text { Hs.Drosha }\end{array}$} & & \\
\hline & $\operatorname{ERL}_{F L G} \mathbf{D}$ & $\mathrm{QRM}_{\mathrm{FLG}} \mathbf{D}_{\ldots \ldots . . .} \mathbf{D}_{\mathrm{LL}} \mathbf{E}$ \\
\hline \multirow{3}{*}{$\begin{array}{c}\text { Dm.Drosha } \\
\text { Ce.Drsh1 } \\
\text { SmDrosha1/2 }\end{array}$} & ERLEFLGD & QRLEFLG \\
\hline & ${ }_{\text {ERLE}} \mathbf{E}_{Y L G} \mathbf{D}$ & $\mathrm{QRL}_{\mathrm{Q} L \mathrm{LG}} \mathbf{D}_{\ldots \ldots \ldots \mathrm{L}} \mathbf{E}$ \\
\hline & ERLEFLGD & QRLEFLG D...... DLLE \\
\hline
\end{tabular}

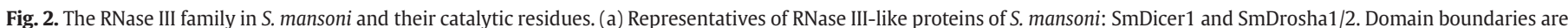

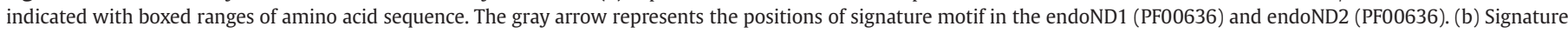

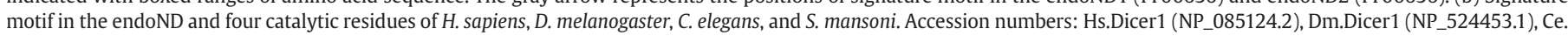

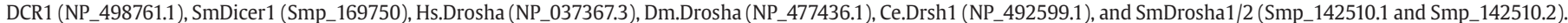




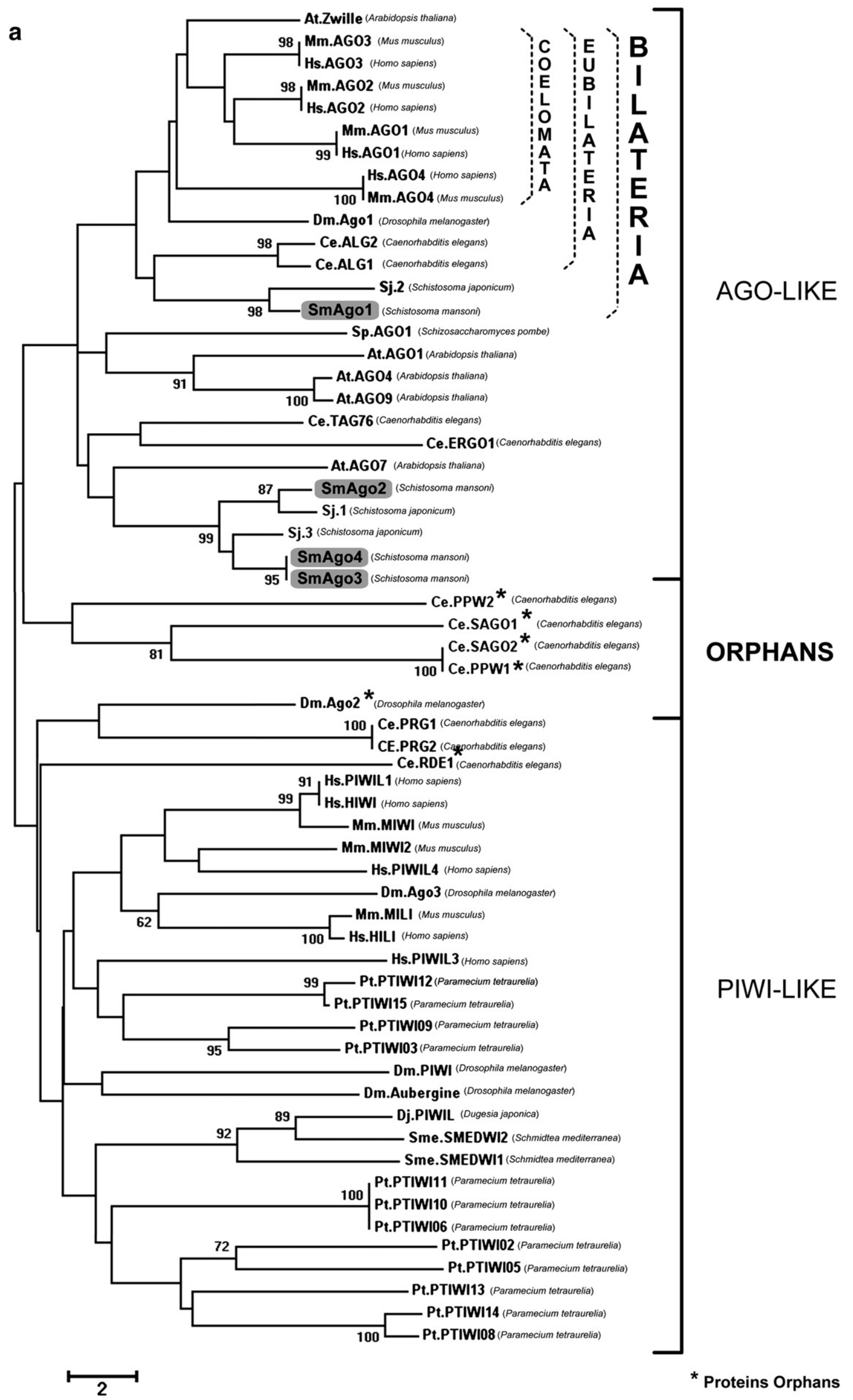




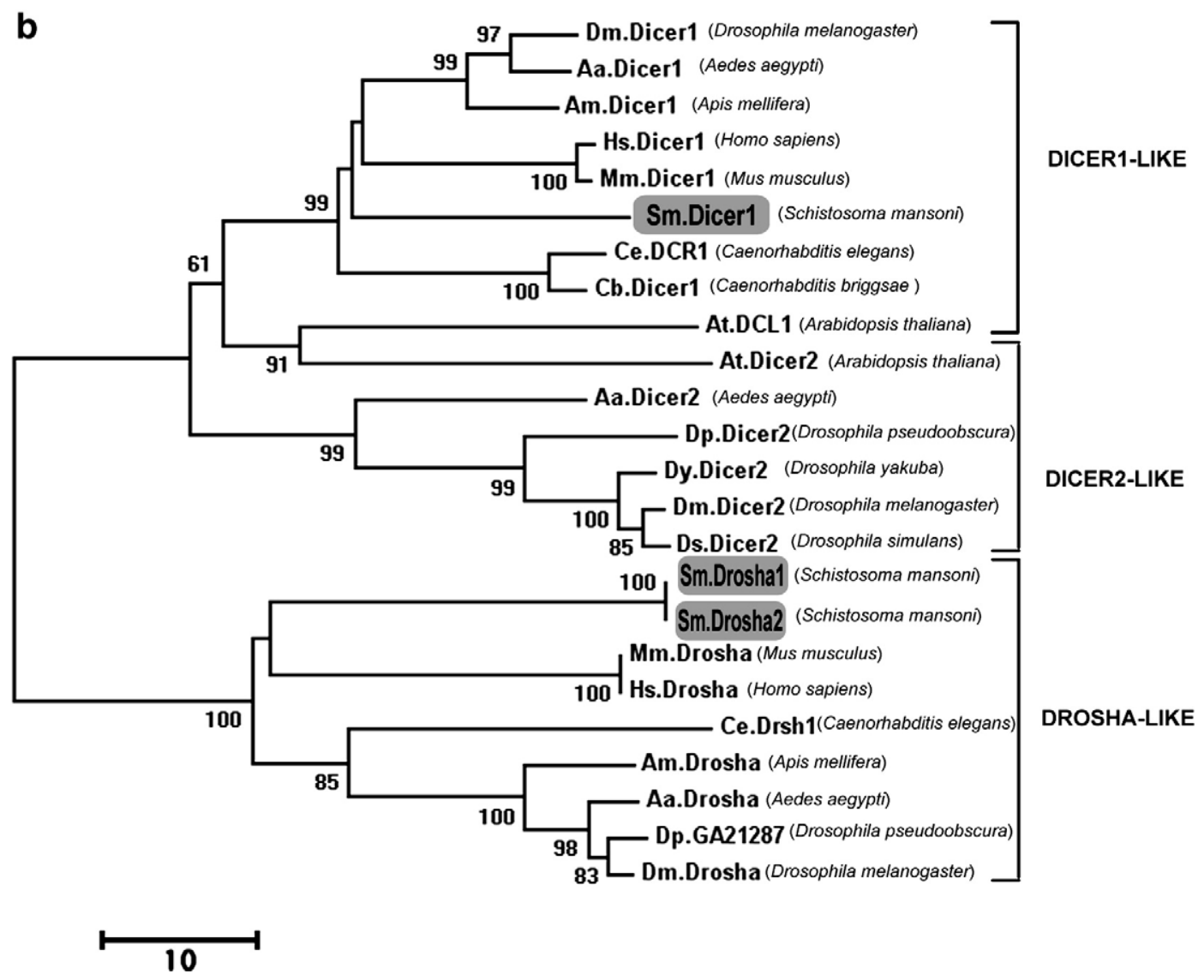

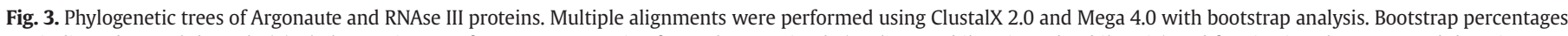

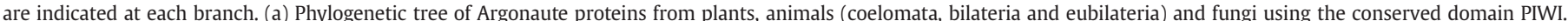

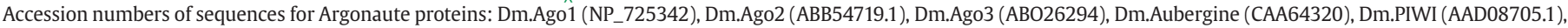

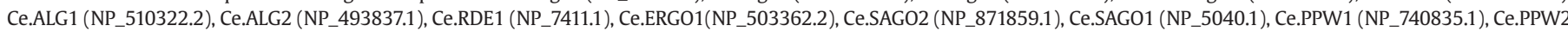

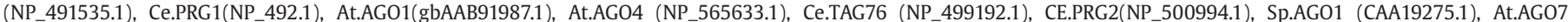

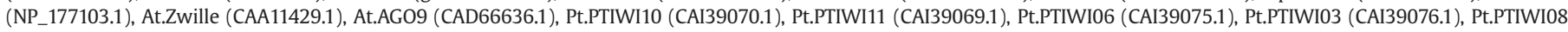

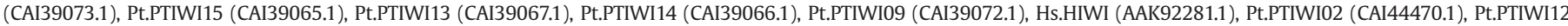

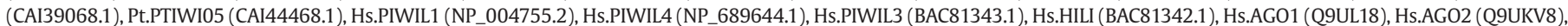

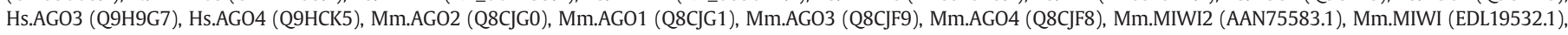

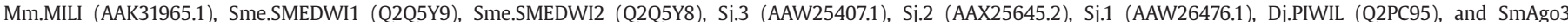

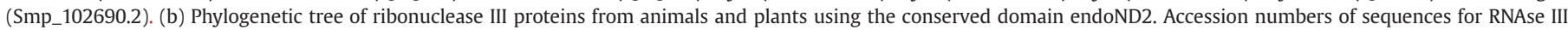

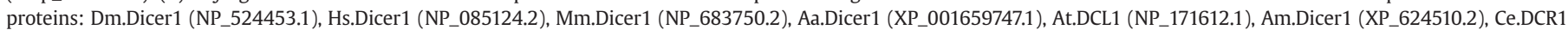

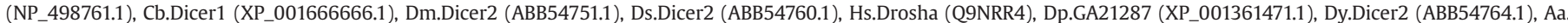

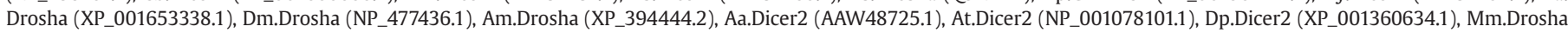
(XP_001473806.1), and Ce.Drsh1 (NP_492599.1).

SmDrosha1/2 proteins in their respective subclades. The phylogenetic relationships of amino acid sequences are shown in Fig. $3 a / b$. The sequences were constituted as an independent branch supported by high bootstrap values (1000 samples). It could be seen that SmAgo1/2/ $3 / 4$ are orthologs of the AGO-like group and are related to C. elegans and $D$. melanogaster orthologs.

Phylogenetic analysis of the RNAse III family in S. mansoni, using the highly conserved endoND domain, revealed the clustering SmDicer1 and SmDrosha1/2 proteins into respective subclades: Dicer1-like and Drosha-like (Fig. 3b).

3.3. Analysis of the relative gene expression profile by quantitative Real Time PCR

We analyzed the expression of SmDicer1 and SmAgo2/3/4 transcripts by relative qRT-PCR in different developmental stages of $S$. mansoni: cercariae, MTS-3.5, MTS-8.5, MTS-18.5, MTS-S24, MTS-48, MTS-72, adult worms and eggs (Fig. 4a/b). Control reactions (where no reverse transcriptase was used) revealed no amplification. Data were normalized relative to an endogenous transcript ( $\mathrm{Sm} \alpha$-tubulin) and were represented as the fold-change in expression relative to the levels found in $24 \mathrm{~h}$ schistosomula (MTS-24) stage. We observed that SmDicer1 and SmAgo2/3/4 transcripts were expressed in all investigated stages and their levels of expression differed significantly $(p<0.001)$ (Fig. 4a/b).

It is worth mentioning that the expression of SmDicer1 increased significantly following the mechanical transformation from cercariae to schistosomula stage (Fig. 4a). For SmAgo2/3/4 transcripts we found a significant decrease from cercariae to MTS-8.5 followed by an increase in MTS-18.5 peaking at MTS-48 stage and then declining in the MTS-72. High and comparable transcript levels for SmAgo2/3/4 were found in the adult and cercariae stages. SmDicer1 and SmAgo2/3/ 4 transcripts reached their high expression levels in the egg stage.

\section{Discussion}

The miRNA pathway consists of a conserved core of proteins and enzymes found in human, $C$. elegans, $D$. melanogaster and $A$. thaliana and known to function in the recognition and processing of dsRNA. dsRNAs are involved in important mechanisms of gene expression and therefore regulate genes involved in the maintenance of the genome integrity [1]. The first evidence of the presence of constituents of the 
miRNA pathway in S. mansoni came from analysis of its transcriptome using the ORESTES strategy [26]. One of the remarkable features of this pathway refers to the fact that miRNAs are small enough to move between cells and sufficiently long to ensure their specificity to the target mRNA. Although several groups have shown that the incorporation of exogenous RNA and its processing into RNAantisense occur in various stages of $S$. mansoni, it is not yet known how the miRNAs contribute to the differential regulation of gene expression [4,27-30].

Through a combination of phylogenetic tree, similarity between domains and mining of the $S$. mansoni database we were able to show putative sequences with conserved domains of the miRNA pathway in this parasite. Our searches have identified thirteen putative sequences that showed significant homology with orthologs of D. melanogaster and $C$. elegans. In addition, there are a number of $S$. japonicum EST sequences displaying a high degree of similarity to components of the miRNA pathway, supporting the existence of this mechanism in Schistosoma spp. During this investigation we were able to verify some species-specific peculiarities, for example, C. elegans does not contain orthologs of Fmr1, Loquacious or Exportin-5. Considering the experimental data that emphasize the importance of the miRNA pathway in C. elegans, the absence of those proteins might be balanced by the existence of others engaged in the same function [31].

In order to understand how closely related the S. mansoni miRNA machinery is compared to orthologs found in model organisms, we selected two key components termed SmAgos and SmDicer for phylogenetic analyses. Using the PIWI domain in the Argonaute

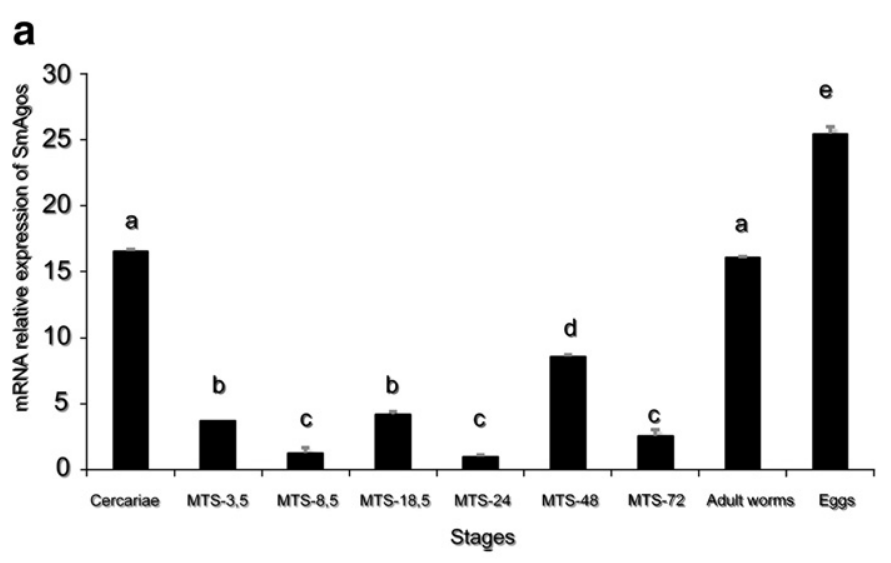

b

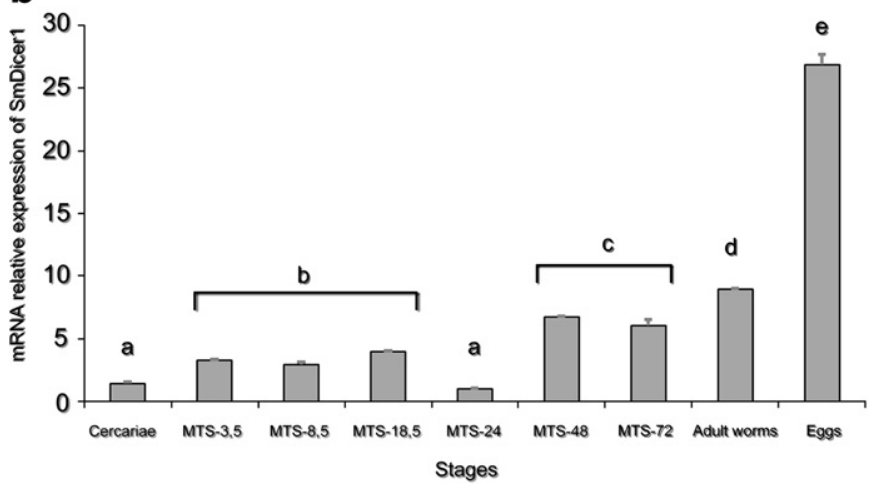

Fig. 4. Developmental expression analysis of S. mansoni (a) SmDicer1 and (b) SmAgo2/3/4. The mRNA expression levels were measured, based on three replicates, in the stages: cercariae, MTS-3.5; MTS-8.5; MTS-18.5; MTS-24; MTS-48, MTS-72 h, adult worms, and eggs using quantitative RT-PCR. Expression levels were calibrated according to the comparative $2^{-\Delta \Delta C t}$ method, using the constitutively expressed $\mathrm{Sm} \alpha$-tubulin as an endogenous control and were normalized relative to the MTS-24 stage. In (a) a, b, c, d and e indicate statistically different expression levels for SmDicer1. In (b) a, b, c, d and e indicate statistically different expression levels for SmAgo2/3/4 (one-way variance analysis followed by Tukey pairwise comparison $p<0.001$ ). proteins it could be seen that these proteins can be divided into two subclades: the AGO-like, based on A. thaliana Ago1 and the PIWI-like, based on $D$. melanogaster Piwi. The PIWI-like and AGO-like exhibit structural differences that may help explain their distinct biological activities. In this work, we have shown that four S. mansoni representatives of Argonautes are AGO-like proteins suggesting their role in the recognition of small RNAs, as a first step towards the silencing of its complementary transcript.

Different Ago proteins have been found in several organisms, ranging from a single member in Schizosaccharomyces pombe, to more than twenty in C. elegans [14,32]. Among the four S. mansoni Argonaute proteins, SmAgo3 and SmAgo 4 are alternative spliced and the same mechanism for generating transcript diversity seems to occur for their orthologs DmAgo1 and CeAlg-2 in Drosophila and C. elegans (FlyBase - http://flybase.bio.indiana.edu/ and WormBase http://www.wormbase.org/).

Alignment of members of the AGO-like revealed that all members of this family, including SmAgo1/2/4, exhibit conservation of the key metal coordinating residues in the PIWI domain known to be responsible for the Slicer activity of Ago proteins. Although the mechanism is far from clear, such activity is the one directly involved in the cleavage of the target mRNA [17,33]. The activity of SmAgos proteins in the regulation of gene expression in different stages of S. mansoni awaits to be demonstrated.

Blaszczyk et al. [43] provided evidence that RNase III family can be divided into four classes. In $D$. melanogaster the fourth class can be separated in 2 types of Dicer as exemplified by Dcr-1 and Dcr-2 [34]. Our phylogenetic analyses revealed that the RNAse III family representatives SmDrosha1/2 and SmDicer could be grouped in their respective categories. The amino acid sequence of these three components showed the conserved endoND domain [35]. However, as shown for other organisms [36], these proteins might operate in different sub-cellular compartments in S. mansoni; SmDrosha1/2 in the nucleus and SmDicer1 in the cytoplasm.

A more detailed investigation of the transcriptome data revealed that ESTs coding for components of the miRNA pathway were sequenced from different stages of S. mansoni (http://verjo18.iq.usp. $\mathrm{br} / \mathrm{schisto} /$ ). Based on observations that the newly transformed schistosomula are characterized by a semi-quiescent state where no net protein synthesis occur $[37,38]$ and the cell-cycle is under arrest [39], we hypothesize that the miRNA pathway may play a role in preventing protein translation, which could favor parasite remodeling at this stage. Blanton and Licate [18] anticipated that the very low protein synthesis at this stage is probably not due to the lack of mRNA, but to a post-transcriptional mechanism that blocks translation. In order to test this hypothesis, we cultivated schistosomula at various time points after transformation and analyzed by qRT-PCR two key transcripts of miRNA pathway, SmAgo2/3/4 and SmDicer1.

Our results confirmed the expression of the SmDicer1 gene in various life-cycle stages of S. mansoni as demonstrated by Krautz-Peterson and Skelly [40]. However, we are the first to investigate its expression in the newly transformed schistosomula. The significant increase in SmDicer1 transcript occurring straight after transformation and its remarkable abundance in eggs suggest an important role in gene silencing at these stages. As a preliminary investigation we decided to perform a global analysis of the expression of SmAgo2/3/4 transcripts in different stages of the $S$. mansoni life-cycle. This allowed us to infer in which stages the miRNA pathway is likely to contribute to gene silencing in the parasite. SmAgo2/3/4 expression was similarly high in cercariae and adult worms and lower in the newly transformed schistosomula. In agreement, a recent proteomic investigation of the $S$. japonicum cercariae secretome revealed the presence of Argonaute-like proteins likely present in high abundance on the cercarial secretions [41]. It seems obvious that Argonaute proteins detected in the secretome were probably not derived from it but contributed as a cytosolic contaminant of the preparation. The high expression levels of SmAgo2/3/4 and SmDicer1 
expression in eggs suggest a role in targeting maternal mRNAs to repress their translation and consequently accelerate their decay as reviewed by Schier [42]. Further experiments are ongoing to test this hypothesis.

Recently, various groups have used the RNAi technique to regulate gene expression in sporocysts, schistosomula, and adult worms [27-30] meaning that gene silencing mechanisms are naturally present on the parasite. Our experiments aimed to uncover a few aspects of the miRNA pathway in S. mansoni; however, several issues still need to be clarified. Of particular importance is to determine the pattern of expression of all components of this pathway, the stage-specific repertoire of miRNA genes and the elucidation of their target transcripts.

\section{Acknowledgments}

We thank the Wellcome Trust Sanger Institute and The Institute for Genomic Research for allowing the use of their sequences that are available via GeneDB, the central data resource for the S. mansoni genome project (http://www.genedb.org/genedb/smansoni/). The $S$. mansoni genome project is funded by the Wellcome Trust and the National Institutes for Health (NIH grant AI48828).

This work was supported by the following Brazilian research agencies: FAPESP (Fundação de Amparo à Pesquisa do Estado de São Paulo), FAPEMIG (Fundação de Amparo à Pesquisa do Estado de Minas Gerais), and CAPES (Coordenação de Apoio ao Pessoal de Nível Superior). We also thank the Malacology Laboratory of CPqRR, Brazil.

\section{References}

[1] He L, Hannon GJ. MicroRNAs: small RNAs with a big role in gene regulation. Nat Rev Genet 2004;5:522-31.

[2] Ambros V. The functions of animal microRNAs. Nature 2004:431:350-5.

[3] Bartel D. MicroRNAs: genomics, biogenesis, mechanism, and function. Cell 2004;116: 281-97.

[4] Meister G, Tuschl T. Mechanisms of gene silencing by double-stranded RNA. Nature 2004:431:343-9.

[5] Gregory RI, Chendrimada TP, Shiekhattar R. MicroRNA biogenesis: isolation and characterization of the microprocessor complex. Methods Mol Biol 2006;342: 33-47.

[6] Song J, Joshua-Tor L. Argonaute and RNA - getting into the groove. Curr Opin Struck Biol 2006;16:5-11.

[7] Lee Y, Ahn C, Han J, Choi H, Kim J, Yim J, et al. The nuclear RNase III Drosha initiates microRNA processing. Nature 2003;425:415-9.

[8] Lund E, Güttinger S, Calado A, Dahlberg JE, Kutay U. Nuclear export of microRNA precursors. Science 2004;303:95-8.

[9] Yi R, Qin Y, Macara IG, Cullen BR. Exportin-5 mediates the nuclear export of premicroRNAs and short hairpin RNAs. Genes Dev 2003;17:3011-6.

[10] Caudy AA, Ketting RF, Hammond SM, Denli AM, Bathoorn AM, Tops BB, et al. A micrococcal nuclease homologue in RNAi effector complexes. Nature 2003;425: 411-4.

[11] Williams RW, Rubin GM. ARGONAUTE1 is required for efficient RNA interference in Drosophila embryos. Proc Natl Acad Sci 2002;99:6889-94.

[12] Sasaki T, Shiohama A, Minoshima S, Shimizu N. Identification of eight members of the Argonaute family in the human genome small star, filled. Genomics 2003;82: 323-30.

[13] Jaronczyk K, Carmichael JB, Hobman TC. Exploring the functions of RNA interference pathway proteins: some functions are more RISCy than others? Biochem J 2005;387:561-71.

[14] Carmell MA, Xuan Z, Zhang MQ Hannon GJ. The Argonaute family: tentacles that reach into RNAi, developmental control, stem cell maintenance, and tumorigenesis. Genes Dev 2002;16:2733-42.
[15] Hutvagner G, Simard MJ. Argonaute proteins: key players in RNA silencing. Nat Rev Mol Cell Biol 2008;9:22-32

[16] Yan KS. Structure and conserved RNA binding of the PAZ domain. Nature 2003;426 (6965):468-74

[17] Song JJ, Smith SK, Hannon GJ, Joshua-Tor L. Crystal structure of Argonaute and its implications for RISC slicer activity. Science 2004;305:1434-7.

[18] Blanton RE, Licate LS. Developmental regulation of protein synthesis in schistosomes. Mol Biochem Parasitol 1992;51:201-8.

[19] Tamura K, Dudley J, Nei M, Kumar S. MEGA4: Molecular Evolutionary Genetics Analysis (MEGA) software version 4.0. Mol Biol Evol 2007;24(8):1596-8.

[20] Saitou N, Nei M. The neighbor-joining method: a new method for reconstructing phylogenetic trees. Mol Biol Evol 1987;4:406-25.

[21] Felsenstein J. Confidence limits on phylogenies: an approach using the bootstrap. Evolution 1985;39:783-91.

[22] Harrop R, Wilson RA. Protein synthesis and release by cultured schistosomula of Schistosoma mansoni. Parasitol 1993;107:265-74.

[23] Basch PF, diConza JJ. In vitro development of Schistosoma mansoni cercariae. J Parasitol 1977;63:245-9.

[24] Webster PJ, Seta KA, Chung SC, Mansour TE. A cDNA encoding an alpha-tubulin from Schistosoma mansoni. Mol Biochem Parasitol 1992;51:169-70.

[25] Livak KJ, Schmittgen TD. Analysis of relative gene expression data using real-time quantitative PCR and the 2(-Delta Delta C(T)) method. Methods 2001;25:402-8.

[26] Verjovski-Almeida S, DeMarco R, Martins EAL, Guimarães PEM, Ojopi EPB, Paquola ACM, Piazza JP, et al. Transcriptome analysis of the acoelomate human parasite Schistosoma mansoni. NatGenet 2003;35:148-57.

[27] Boyle JP, Wu XJ, Shoemaker CB, Yoshino TP. Using RNA interference to manipulate endogenous gene expression in Schistosoma mansoni sporocysts. Mol Biochem Parasitol 2003;128:205-15

[28] Skelly PJ, Dadara A, Harn DA. Suppression of cathepsin B expression in Schistosoma mansoni by RNA interference. Int J Parasitol 2003;33:363-9.

[29] Dinguirard N, Yoshino TP. Potential role of a CD36-like class B scavenger receptor in the binding of modified low-density lipoprotein (acLDL) to the tegumental surface of Schistosoma mansoni sporocysts. Mol Biochem Parasitol 2006;146:219-30

[30] Osman A, Niles EG, Verjovski-Almeida S, LoVerde PT. Schistosoma mansoni TGFbeta receptor II: role in host ligand-induced regulation of a schistosome target gene. PLoS Pathog 2006;2:536-50.

[31] Ouellet DL, Perron MP, Gobeil L, Plante P, Provost P. MicroRNAs in gene regulation: when the smallest governs it all. J Biomed Biotechnol 2006;69616:1-20.

[32] Yigit E, Batista PJ, Bei Y, Pang KM, Chen CG, Tolia NH, et al. Analysis of the C. elegans Argonaute family reveals that distinct Argonautes act sequentially during RNAi. Cell 2006;127:747-57.

[33] Tolia NH, Joshua-Tor L. Slicer and the Argonautes. Nat Chem Biol 2007:3:36-43.

[34] Tijsterman M, Plasterk RH. Dicers at RISC; the mechanism of RNAi. Cell 2004;117: $1-3$.

[35] Ji X. Structural basis for non-catalytic and catalytic activities of ribonuclease III. Acta Crystallogr 2006;D62:933-40.

[36] Gan J, Shaw G, Tropea JE, Waugh DS, Court DL, Ji X. A stepwise model for doublestranded RNA processing by ribonuclease III. Mol Microbiol 2008;67:143-54.

[37] Nagai Y, Gazzinelli G, de Moraes GW, Pellegrino J. Protein synthesis during cercaria-schistosomulum transformation and early development of the Schistosoma mansoni larvae. Comp Biochem Physiol B 1977;57:27-30.

[38] Lawson JR, Wilson RA. Metabolic changes associated with the migration of the schistosomulum of Schistosoma mansoni in the mammal host. Parasitol 1980:81: 325-36.

[39] Clegg JA. In vitro cultivation of Schistosoma mansoni. Exp Parasitol 1965;16: 133-47.

[40] Krautz-Peterson G, Skelly PJ. Schistosoma mansoni: the dicer gene and its expression. Exp Parasitol 2008;117:337-47.

[41] Dvorak J, Mashiyama ST, Braschi S, Sajid M, Knudsen GM, Hansell E, et al Differential use of protease families for invasion by schistosome cercariae. Biochimie 2008;90:345-58.

[42] Schier AF. The maternal-zygotic transition: death and birth of RNAs. Science 2007;316:406-7.

[43] Blaszczyk J, Gan J, Tropea JE, Court DL, Waugh DS, Ji X. Noncatalytic assembly of ribonuclease III with double-stranded RNA. Structure 2004;12:457-66. 